\title{
A Comparison Between Local Wave Speed in the Carotid and Femoral Arteries in Healthy Humans: Application of a New Method
}

\author{
Alessandra Borlotti, Sebastian Vermeersch, Ernst Rietzschel, Patrick Segers, Ashraf W. Khir
}

\begin{abstract}
The wave speed (c) and the arrival time of reflected wave $(T r w)$ in the common left carotid artery and common left femoral artery have been evaluated in $\mathbf{7 0}$ healthy subjects, aged 35-55 years with a non-invasive method.

Wave speed and the arrival time of reflected waves were determined with InDU-loop and non-invasive wave intensity analysis $\left(_{n}\right.$ dI) techniques, respectively. Diameter (D) was measured with ultrasound echo wall tracking and velocity (U) was obtained by ultrasonography. A statistical analysis has been carried out in order to establish a potential relation of $c$ and $T r w$ with gender and age in the study population. Subjects have been divided in two classes of age, one from 35 to 45 years and the other from 45 to 55 years.

Results show that $c$ and $T r w$ in the femoral artery are higher than those in carotid, in both men and women $(P<0.001)$. Also, the distance of the reflection ( $L$ ) site from the point of measurement is higher in the femoral than in the carotid artery. We did not find statistically significant differences between $c$, age or gender in femoral artery. However, $c$ in the carotid artery increases with age $(P<0.05)$, but did not change between men and women.

In this paper InDU-loop has been used for the first time to determine $c$ and Trw in carotid and femoral arteries in a large population of healthy subjects.
\end{abstract}

\section{INTRODUCTION}

$\mathrm{T}$ HE local wave speed in arteries can be used as an indicator of arterial stiffness [1,2], with increased arterial stiffness being considered as an early phenotype of atherosclerosis and potential prognostic factor when assessing the individual's total cardiovascular risk. In the past few decades several methods to determine this mechanical parameter in real time have been developed. The most common is the foot-to-foot method, which is based on the measurements of pressure or velocity at two sites located at a known distance and the determination of the time delay between the two measurements.

For one-point measurements, Westerhof et al [3] assumed that characteristic impedance could be an indicator of wave

Manuscript received April 1, 2010.

Alessandra Borlotti, Brunel Institute for Bioengineering, Brunel University, Greater London, UK alessandra.borlotti@brunel.ac.uk

Sebastian Vermeersch, Ghent University, IBiTech-bioMMeda, Gent, Belgium Sebastian.Vermeersch@ugent.be

Ernst Rietzschel, Ghent University Hospital, Gent, Belgium Ernst.Rietzschel@ugent.be

Patrick Segers, Ghent University, IBiTech-bioMMeda, Gent, Belgium Patrick.Segers@ugent.be

Ashraf W. Khir, Brunel Institute for Bioengineering, Brunel University, Greater London, UK Ashraf.Khir@brunel.ac.uk speed. Khir et al. [4] introduced the PU-loop method (with P pressure and $U$ velocity) that consists of the determination of the slope of the linear portion of the loop that equals $\rho c$, where $\rho$ is blood density. Davies et al [5] determined wave speed in coronary arteries minimizing the net wave energy. These approaches, however, require the measurement of local pressure, which is difficult to measure noninvasively. More recently, Feng and Khir [6] developed a new method based on noninvasive and simultaneous measurements of diameter and flow velocity to determine local wave speed and to separate diameter, velocity and wave intensity waveforms into their forward and backward components. Local wave speed is determined from the slope of the linear portion of $\ln$ DU-loop (with $\mathrm{D}$ diameter), which is equal to $1 / 2$ $c$. While the reliability of this method has been assessed in laboratory hydraulic bench experiments, the applicability in a clinical setting and using routine clinical measuring equipment has not been demonstrated.

The aims of this study are therefore 1) determine local wave speed in carotid and femoral arteries in a population of 70 healthy subjects using lnDU-loop 2) determine the arrival time of reflected waves and the distance of the reflection site by means of the non-invasive wave intensity analysis, and 3) examine $c$ and $\operatorname{Tr} w$ as function of age and gender.

\section{THEORETICAL CONSIDERATIONS}

\section{A. Determination of wave speed from velocity and} diameter

It is well known that wave speed is a function of the distensibility of the tube wall and the density of fluid.

$c^{2}=\frac{A d P}{\rho d A}$

where $\mathrm{A}$ is the initial cross-sectional area and $\mathrm{dA}$ its change. Re-arranging equation (1), we can write

$d P=\rho c^{2} \frac{2 d D}{D}$

where $\mathrm{D}$ is the diameter and $\mathrm{dD}$ its change.

The change in pressure $\mathrm{dP}$ is the sum of the forward and backward pressure waveforms and we can reasonably consider that this relation is also valid for the diameter change

$d D=d D_{+}+d D_{-}$

Substituting (3) into (2) 
$d P_{+}+d P_{-}=\frac{2 \rho c^{2}}{D}\left(d D_{+}+d D_{-}\right)$

Recalling the water hammer equation

$$
d P_{ \pm}=\rho c d U_{ \pm}
$$

and assuming that $\mathrm{dU}$ equals the algebraic summation of the change in forward and backward directions, we can write

$c=\frac{D}{2} \frac{\left(d U_{+}+d U_{-}\right)}{\left(d D_{+}+d D_{-}\right)}$

Considering $\frac{d D}{D}=d \ln D$, the wave speed can be determined as,

$c= \pm \frac{1}{2} \frac{d U_{ \pm}}{d \ln D_{ \pm}}$

In the $\operatorname{lnD}-\mathrm{U}$ plot we can identify by eye a linear portion of the loop in the early part of the systole; its slope equals $1 / 2 c$.

\section{B. Noninvasive wave intensity analysis}

A noninvasive wave intensity can be calculated from diameter and velocity as ${ }_{n} \mathrm{dI}=\mathrm{dDdU}$ [6]. Separating in forward and backward directions and re-arranging equation (6), ${ }_{n} \mathrm{dI}$ can be written as

${ }_{n} d I_{ \pm}=\frac{1}{4(D / 2 c)}\left(d D \pm \frac{D}{2 c} d U_{ \pm}\right)^{2}$

Non invasive wave intensity analysis has the same useful characteristic of the traditional analysis, being positive for forward waves and negative for backward waves. The arrival time of the reflected waves (Trw) that is the time that it took the wave to travel from the site of measurements, be reflected and came back can be determined as the difference between the onset of the forward wave and the onset of the backward one. The distance (L) of the reflection site from the site of measurements can be calculated as $L=\frac{c \operatorname{Tr} w}{2}$.

\section{Methodology}

\section{A. Study population}

The study population comprised 70 subjects ( 35 men and 35 women) aged 35-55 years, free from overt cardiovascular disease. This population represents a subgroup of the Asklepios study, a longitudinal population study designed to focus on the interplay between aging and cardiovascular haemodynamics [7].

\section{B. Measurement of local arterial diameter}

Arterial diameter distension waveforms were obtained by ultrasound echo wall tracking performed at the left common carotid artery and at the left common femoral artery using an ultrasonographic system (Vivid7; GE Vingmed, Horten,
Norway). During this procedure, vessel boundary is selected manually at a given moment in time and its movement is automatically tracked using a modified autocorrelation estimator. In this study the outer vessel diameter was detected by tracking the media-adventitia boundary on both the anterior and posterior walls $[7,8,9]$.

\section{Measurement of arterial velocity}

Arterial flow velocities, in left common carotid artery and left common femoral artery, were obtained using a commercially available ultrasonographic system [7]. Images, in DICOM format, were processed offline within a dedicated software written in Matlab (The Mathworks Inc, Natick, Massachusetts, USA). After a preliminary filtering of the images, a morphological operation derived from a combination of the fundamental morphological operations dilation and erosion was applied in order to smooth image contours. Then, the maximum and minimum velocity envelope (Fig.1) was detected and the average profile was used to calculate the wave speed by means of lnDU-loop (7).

Since $U$ and D were not recorded simultaneously, they were aligned by means of ECG. R-peaks were detected and heart rate was calculated for each beat. D and $\mathrm{U}$ cycles with the same heart rate were used to determine $c$. Simultaneous measurements of $\mathrm{U}$ and $\mathrm{D}$ is important in $c$ determination, but in our case the interval time between the two recordings is very short that we can assume that haemodynamics parameters did not alter significantly.

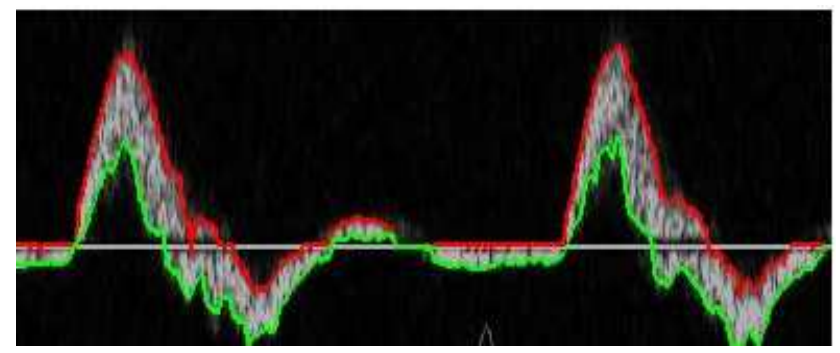

Fig.1. Detection of maximum and minimum velocity envelope in DICOM image.

\section{Statistical analysis}

Data are presented as mean values \pm SD. Comparison between carotid and femoral arteries for $c$ and Trw, male and female groups for $c$ and age groups for $c$ were assessed by paired Student t-test. P values less than 0.05 were considered statistically significant. Statistical analyses were performed using SPSS 15.0 (SPSS Inc., Chicago, Illinois, USA).

\section{RESULTS}

Figure 2 show two examples of $\operatorname{lnDU}-1$ loop at the carotid and femoral arteries. The initial portion of the loop, when it is most probable that only forward waves are present, is clearly linear. Wave speed calculated from $\ln D U$-loop slope is $4.42 \mathrm{~m} / \mathrm{s}$ and $5.16 \mathrm{~m} / \mathrm{s}$ for carotid and femoral arteries 
respectively.

Figure 3 and Figure 4 show two examples of a D and $U$ waveform (a) and noninvasive wave intensity analysis (b) for a carotid and femoral artery, respectively. The arrow indicates the arrival time of reflected waves. We can identify three peaks corresponding to: a forward compression wave, a backward compression wave and a forward expansion wave. The same peaks can be found in the traditional wave intensity analysis.

In Table 1, the average values of wave speed and arrival time of reflected waves by sex in the study population are reported.

Dividing subjects into two classes of age (35-45 and 45-55 years) we found that $c$ determined using $\ln D U-l o o p$ does not vary significantly with age in the femoral artery $(5.19 \mathrm{~m} / \mathrm{s} \pm$ $2.20 \mathrm{~m} / \mathrm{s}$ for the $35-45$ group and $5.4 \mathrm{~m} / \mathrm{s} \pm 2.00 \mathrm{~m} / \mathrm{s}$ for the 45-55 group) and increases by about $15 \%$ in carotid artery $(3.82 \mathrm{~m} / \mathrm{s} \pm 0.99 \mathrm{~m} / \mathrm{s}$ for $35-45$ group and $4.4 \mathrm{~m} / \mathrm{s} \pm 1.29 \mathrm{~m} / \mathrm{s}$ for $45-55$ group, $\mathrm{P}<0.05)$. We did not find any statistical significant dependence of wave speed on gender.

(a)

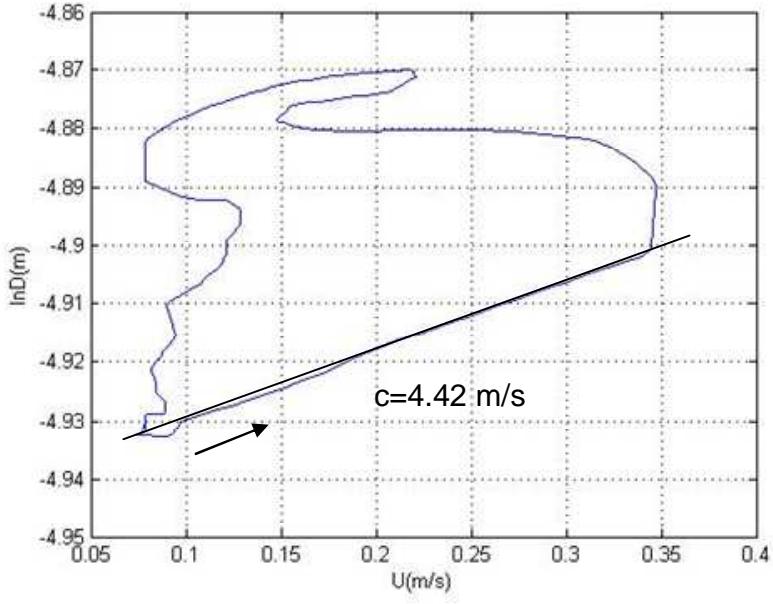

(b)

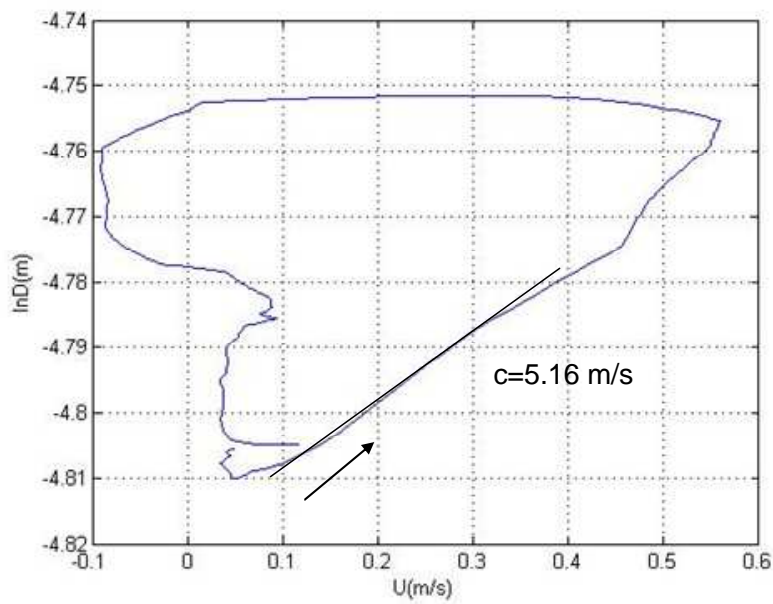

Fig.2. Wave speed determined in carotid artery (a) and femoral artery (b) by lnDU-loop in a female aged 43. Black lines identify the linear portion of the loop and the arrows their direction.
Table 1. Average values of age, mean blood pressure, carotid and femoral $c$ Trw and $\mathrm{L}$ in male and female.

\begin{tabular}{ccc}
\hline & MALE & FEMALE \\
& $\mathrm{N}=35$ & $\mathrm{~N}=35$ \\
\hline Age \pm SD & $48 \pm 5$ & $45 \pm 5$ \\
Mean arterial & & \\
pressure (mmHg) & $99 \pm 11$ & $104 \pm 13$ \\
$\pm \mathrm{SD}$ & & \\
$c$ car $(\mathrm{m} / \mathrm{s}) \pm \mathrm{SD}$ & $4.16 \pm 1.58$ & $3.71 \pm 1.21$ \\
$c$ fem $(\mathrm{m} / \mathrm{s}) \pm \mathrm{SD}$ & $5.42 \pm 2.16$ & $5.64 \pm 2.19$ \\
$\operatorname{Tr} w$ car $(\mathrm{s}) \pm \mathrm{SD}$ & $0.036 \pm 0.014$ & $0.035 \pm 0.016$ \\
$T r w$ fem $(\mathrm{s}) \pm \mathrm{SD}$ & $0.089 \pm 0.028$ & $0.075 \pm 0.034$ \\
$\mathrm{~L}$ car $(\mathrm{cm}) \pm \mathrm{SD}$ & $6.9 \pm 2.8$ & $6.3 \pm 3$ \\
$\mathrm{~L} \mathrm{fem}(\mathrm{cm}) \pm \mathrm{SD}$ & $23.3 \pm 10$ & $20.6 \pm 11.2$ \\
\hline
\end{tabular}

\section{DISCUSSION}

The lnDU-loop [6] allows the noninvasive assessment of wave speed; for this reason it can be used as an advantageous procedure in clinical environment. In the present work this method has been applied to determine the wave speed in the left common carotid and left common femoral artery, within a population of 70 healthy subjects.

We found that the wave speed in the femoral artery is on average higher than in the carotid artery $(\mathrm{P}<0.001)$ which is in agreement with previous findings by other investigators [10].

Vermeersch et al [11] derived, for the whole population of the Asklepios study, local carotid and femoral wave speed from the distensibility coefficient using the Bramwell-Hill equation:

$$
c=\sqrt{\frac{1}{\rho D_{S}}}
$$

where $D_{S}$ is the distensibilty and $\rho$ is the density of blood. They found that carotid wave speed increases with age regardless of gender, and that there is not a significant difference between men and women. With regard to femoral wave speed they identified a higher value in men than in women. Apart from the latter finding, the results of this small prospective feasibility study appear to be in line with results reported by Vermeersch et al. for the complete population.

In this paper the arrival time of reflected waves and the distance from the reflection site have been determined by noninvasive wave intensity analysis. Trw in the femoral artery is higher than $T r w$ in carotid artery $(\mathrm{P}<0.001)$. The distances from the reflection site, derived from $\operatorname{Tr} w$ and $c$, are $6.9 \mathrm{~cm} \pm 2.8 \mathrm{~cm}$ and $6.3 \mathrm{~cm} \pm 3 \mathrm{~cm}$ for the carotid artery in men and women respectively and $23.3 \mathrm{~cm} \pm 10 \mathrm{~cm}$ and $20.6 \mathrm{~cm} \pm 11.2 \mathrm{~cm}$ for the femoral artery in men and women respectively. 
(a)
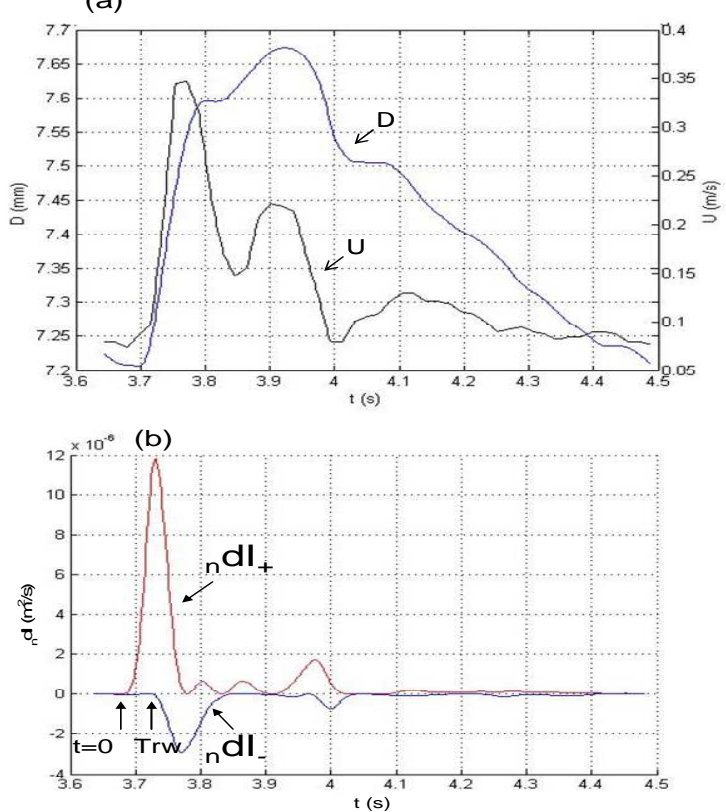

Fig.3. D and U waveforms (a) and ${ }_{n} \mathrm{dI}$ separated in its forward and backward components (b) in carotid artery. The arrows indicate the onset of the forward wave $(\mathrm{t}=0)$ and the onset of the backward wave $(\operatorname{Tr} w=0.05 \mathrm{~s})$.

\section{LIMITATIONS}

Determination of $c$ by means of $1 n$ DU-loop could be affected by errors due to (i) the detection of its linear portion by eye, in this case the inter- and intra- observer variability should be investigated and (ii) the fact that $U$ and $D$ were not recorded at the same time.

\section{CONCLUSION}

The lnDU-loop and noninvasive wave intensity analysis are useful and reliable methods to determine wave speed and arrival time of reflected waves. In order to develop a more accurate statistical analysis, the study should involve the entire Asklepios population.
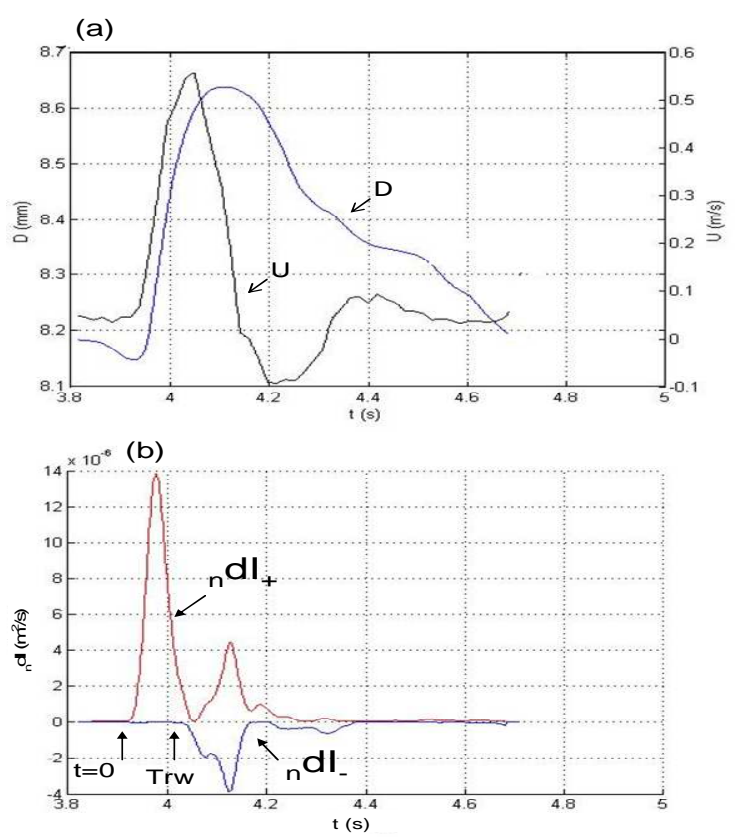

Fig.4. D and $U$ waveforms (a) and ${ }_{n} \mathrm{dI}$ separated in its forward and backward components (b) in femoral artery. The arrows indicate the onset of the forward wave $(\mathrm{t}=0)$ and the onset of the backward wave $(\operatorname{Tr} w=0.12 \mathrm{~s})$.

\section{REFERENCES}

[1] J. Blacher, R. Asmar, S. Djane, "Aortic pulse wave velocity as a marker of cardiovascular risk in hypertensive patients". Hypertension vol. 33, pp. 1111-1117, 1999.

[2] S. Laurent, P. Boutouyrie, R. Asmar, I. Gautier, B. Laloux, L. Guize, P. Ducimetiere, Benetos, "Aortic stiffness is an independent predictor of all-cause and cardiovascular mortality in hypertensive patients". Hypertension vol. 37, pp. 1236-1241, 2001.

[3] N. Westerhof, P. Sipkema, GC Van Den Bos, G. Elzinga, "Forward and backward waves in the arterial system". Cardiovascular Research vol. 6(6), pp. 648-656, 1972.

[4] A.W. Khir, A. O'Brien, S. Gibs, K.H. Parker, "Determination of wave speed and wave separation in the arteries". Journal of Biomechanics vol. 34 (9), pp. 1145-1155, 2001.

[5] J.E. Davies, Z.I. Whinnett, D.P. Francis, K. Willson, R.A. Foale, I.S Malik, A.D. Hughes, K.H. Parker, J. Mayet "Use of simultaneous pressure and velocity measurements to estimate arterial wave speed at a single site in human”. American Journal of Physiology: Heart and Circulatory Physiology vol. 290(2), pp. 878-885, 2006.

[6] J. Feng and A.W. Khir, "Determination of wave speed and wave separation in the arteries using diameter and velocity". Journal of Biomechanics vol. 43(3), pp. 455-462, 2010.

[7] E.R. Rietzschel, M.L. De Buyzere, S. Bekaert, P. Segers, D. De Bacquer, L. Cooman et al. "Rational, design, method and baseline characteristics of the Asklepios study". European Journal of Cardiovascular Prevention and Rehabilitation 14, pp.179-191, 2007.

[8] P. Segers, S.I. Rabben, J. De Backer, J. De Sutter, T.C. Gillebert, L. Van Bortel, P. Verdonk, "Functional analysis of the common carotid artery: relative distension differences over the vessel wall measured in vivo". Journal of Hypertension vol. 22(5), pp. 973-981, 2004.

[9] S.I. Rabben, S. Baerum, V. Sorhus, H. Torp, "Ultrasound-based vessel wall tracking: an auto-correlation technique with RF center frequency estimation". Ultrasound Med Biol. vol. 28(4), pp. 507-17, 2002.

[10] D.A. McDonald, "Blood flow in arteries" 1974, pp. 389-419.

[11] S.J. Vermeersch, E.R. Rietzschel, M.L. De Buyzere, D. De Bacquer, G De Backer, L.M. Van Bortel, T.C. Gillebert, P. et al. "Age and gender related patterns in carotid-femoral PWV and carotid and femoral stiffness in a large healthy, middle-aged population". Journal of Hypertension vol. 26, pp. 1411-1419, 2008. 